\title{
Stress and Strain on the Future of Nuclear Cardiology
}

\author{
Sarv Priya* and Michael M Graham \\ University of Iowa Hospitals \& Clinics, USA
}

*Corresponding author: Sarv Priya, Division of Nuclear Medicine, Department of Radiology, University of Iowa Hospitals \& Clinics, Iowa, USA, 52242.

Received Date: August 16, 2018

Published Date: August 24, 2018

\section{Editorial}

According to the Centers for Disease Control and Prevention, heart disease and cancer are the two major causes of mortality. Coronary artery disease is the most common cause for mortality related to heart disease, while in oncology, prostate cancer is one of the leading causes of death among men of all races [1]. It is important that we detect primary and recurrent cardiac and malignant disease early and thus provide timely treatment to the patients, increasing thereby the chances of their survival. In recent years there has been tremendous growth in the field of cardiovascular imaging both for diagnosis and prognostication. A major methodology for risk stratification that has been in place for many years now is cardiac Single Photon Emission Computed Tomography (SPECT). It is an excellent tool for myocardial perfusion imaging, facilitating timely management decisions regarding revascularization. The test has been validated repeatedly and its efficacy has been proven for quite a long time.

More recently, cardiac Positron Emission Tomography (PET) has shown additional advantages over SPECT. These include higher detection efficiency, increased temporal and spatial resolution, shorter acquisition time, improved attenuation correction and reduced radiation dose [2]. A particularly important advantage of cardiac PET is its ability to quantify absolute myocardial blood Flow (MBF) and myocardial flow reserve (MFR). Recent literature has advocated the utility of MBF and MFR in improved riskstratification of coronary heart disease patients [3]. In comparison to SPECT, PET perfusion imaging with MBF and MFR quantification results in increased detection of severe obstructive coronary artery disease and increased revascularization rate [4].

However, despite its advantages, PET has not become universal in usage. This is likely because of its relatively higher cost and the short half-life of available radiotracers that require generators or on-site cyclotrons. The short half-life of commonly used radiotracers also makes exercise perfusion imaging challenging with $13 \mathrm{~N}$-Ammonia ( $\mathrm{t} 1 / 2$ of 10 minutes) and virtually impossible with 82 Rubidium (t1/2 of 1.25 minutes) [5].
Another reason for the lower usage of cardiac PET is the competition for PET scanner time with other specialties, primarily oncology. This is a significant problem in many academic or private centers (small as well as large), which do not have a dedicated PET scanner for cardiac patients.

At the same time, detection of metastases in patients with suspected recurrence of prostate cancer with rising prostate specific antigen (PSA) levels is an area of ongoing research. Currently, Tc-99 MDP bone scan and 18F-Sodium Fluoride PET are the mainstay of detection of metastases in prostate cancer. Recently, particularly in Europe, there has been an increase in the number of studies with a novel PET agent, Ga-68 PSMA (prostate specific membrane antigen). PSMA has been shown to detect metastases even if PSA level is $<0.5 \mathrm{ng} / \mathrm{ml}[6,7]$. Studies have reported significant changes in treatment decisions taken on PSMA based PET scanning [8]. PSMA based imaging has been shown superior to other widely available C-11 choline and F-18 fluoromethylcholine, especially at low PSA levels [9]. The earlier detection of metastases may result in improved outcomes. PSMA based imaging is currently in Phase III trial in Unites States and is likely to get FDA approval in the next 3-4 years.

Cardiac PET perfusion imaging is also set to take a giant leap by the introduction of novel agent F-18 Flurpiridaz that is currently in Phase III trial and is likely to receive FDA approval much sooner. This agent has added advantages over N-13 Ammonia and Rubidium-82 as it has a higher extraction fraction (almost approximating 0-15 Water), longer half-life of 109 minutes, higher spatial resolution (due to shorter positron range), and greater availability via a regional distribution similar to that for FDG5. This will make it possible to employ F-18 Flurpiridaz in all centers that have PET scanner. In addition, physiological exercise-based perfusion imaging is also possible because of its long half-life. Recent clinical trials are moving towards adding MBF and MFR to determine the physiological significance of coronary artery disease. This approach will also be useful in the detection of microvascular disease as well 
as balanced ischemia by using dynamic PET imaging.

Currently, SPECT perfusion imaging is limited in terms of detection of microvascular disease. FDA approval of F-18 Flurpiridaz will make this drug widely available and this will increase the need for more myocardial perfusion imaging using PET.

The net impact of all these advancements will be a marked increase in demand for PET-CT imaging for more accurate diagnosis of coronary artery disease and also the timely treatment of prostate cancer. In a world becoming increasingly "Google oriented" the patients will demand the best test, and this is likely to result in a markedly increased demand for PET imaging.

Currently many PET centers are already operating near capacity with significant wait times. The popularization of these new agents is likely to cause even longer wait times for PET imaging. SPECT perfusion imaging will continue to be used; although it may not be as preferable as the PET it will be readily available. It is likely that in some settings, such as pre-operative screening, SPECT imaging may be as effective as PET, although this will need to be determined in clinical trial or careful retrospective studies.

There are a limited number of PET scanners currently in use and a marked demand for PET imaging services is going to occur in the next few years. It is important to recognize and acknowledge this situation well ahead of time and take remedial measures. For that the need is to enhance the facilities, hire additional personnel, and improve scheduling in a timely manner, or there will be large numbers of frustrated and angry patients, cardiologists, oncologists, radiologists, and nuclear medicine physicians.

\section{References}

1. National Center for Health Statistics (2017) Health, United States, 2016: with chartbook on long-term tends in health. Hyattsville.
2. Humm JL, Rosenfeld A, Del Guerra A (2003) From PET detectors to PET scanners. Eur J Nucl Med Mol Imaging 30(11): 1574-1597.

3. Ziadi MC (2017) Myocardial flow reserve (MFR) with positron emission tomography (PET)/computed tomography (CT): clinical impact in diagnosis and prognosis. Cardiovasc Diagn Ther 7(2): 206-218.

4. Knight S, Min DB, Le VT, Meredith KG, Dhar R (2018) Implementation of a cardiac PET stress program: comparison of outcomes to the preceding SPECT era. JCI Insight 3(9).

5. Di Carli MF, Murthy VL (2011) Cardiac PET/CT for the evaluation of known or suspected coronary artery disease. Radiographics 31(5): 1239-1254

6. Cher ML, Bianco FJ Jr, Lam JS, Davis LP, Grignon DJ (1998) Limited role of radionuclide bone scintigraphy in patients with prostate specific antigen elevations after radical prostatectomy. J Urol 160(4): 1387-1391.

7. Crawford ED, Koo PJ, Shore N, Slovin SF, Concepcion RS (2018) A clinician's guide to next generation Imaging in Patients With Advanced Prostate Cancer (Prostate Cancer Radiographic Assessments for Detection of Advanced Recurrence [RADAR] III). J Urol (18): 4364343649.

8. Emmett L, van Leeuwen PJ, Nandurkar R, Scheltema MJ, Cusick T (2017) Treatment outcomes from ${ }^{68} \mathrm{Ga}$-PSMA PET/CT-informed salvage radiation treatment in men with rising PSA after radical prostatectomy: prognostic value of a negative PSMA PET. J Nucl Med 58(12): 1972-1976.

9. Morigi JJ, Stricker PD, van Leeuwen PJ, Tang R, Ho B (2015) Prospective comparison of $18 \mathrm{~F}$-fluoromethylcholine versus 68Ga-PSMA PET/CT in prostate cancer patients who have rising PSA after curative treatment and aAre bBeing considered for targeted Ttherapy. J Nucl Med 56(8): $1185-1190$ 